\title{
Status of Measles Elimination in Eleven Countries with High Routine Immunisation Coverage in The WHO African Region
}

\author{
Balcha Masresha ${ }^{1 *}$, Richard Luce ${ }^{2}$, Messeret Shibeshi ${ }^{3}$ Reggis Katsande' ${ }^{1}$ Amadou Fall ${ }^{4}$, Joseph Okeibunor', \\ Goitom Weldegebriel ${ }^{3}$, Richard Mihigo' \\ 'WHO Regional Office for Africa, Brazzaville, Congo \\ ${ }^{2}$ WHO Inter-country Support Team for Central Africa, Libreville, Gabon \\ ${ }^{3}$ WHO Inter-country Support Team for East and Southern Africa, Harare, Zimbabwe \\ ${ }^{4}$ WHO Inter-country Support Team for Western Africa, Ouagadougou, Burkina Faso
}

Article Info

\section{Article Notes}

Published: August 02, 2018

\section{*Correspondence:}

Dr. Balcha G Masresha, WHO Regional Office for Africa,

Brazzaville, Congo; Telephone No: +263 77503 5369;

Email: masreshab@who.int.

(c) 2018 Masresha BG. This article is distributed under the terms of the Creative Commons Attribution 4.0 International License.

\section{Keywords:}

Measles Elimination

Immunization

Coverage

Status

WHO African Region

\section{ABSTRACT}

Background: Measles elimination is defined as the absence of endemic measles virus transmission in a defined geographic area for at least 12 months in the presence of a well-performing surveillance system. The WHO framework for verification of measles elimination indicates that the achievement of measles and/or rubella elimination should be verified for individual countries.

Objective: We identified 11 high performing countries based on their first dose measles vaccination coverage, and looked at their performance across the various programmatic parameters, to see if they are ready to undertake the verification of measles elimination.

Methods: We identified 11 countries with $>90 \%$ measles first dose coverage for the most recent 5 years according to the WHO UNICEF estimates of national immunisation coverage. We analysed vaccination coverage and surveillance performance in these countries.

Results: Algeria, Botswana, Gambia, Mauritius, Rwanda, Seychelles have maintained measles first dose (MCV1) coverage of 95\% or more since 2011. In 2015, only Algeria, Cape Verde and Seychelles had coverage of $95 \%$ or more for the second dose of measles vaccine (MCV2). Of the 22 supplemental immunisation activities (SIAs) among the 11 countries, only 6 had administrative coverage of less than 95\%. Only Rwanda and Lesotho attained the case-based surveillance performance targets in all the five years.

Conclusion: Despite their high routine first dose measles immunisation coverage, all of the 11 countries have some program gaps indicating that they do not meet all the criteria to undergo verification of elimination at this point. It is recommended for these countries to set up national verification committees as per the WHO framework for verification of measles elimination, in order to initiate the documentation and monitoring of progress, and to address programmatic gaps in the coming years.

\section{Introduction}

The attainment of sustained high population immunity through high levels of measles vaccination coverage in the routine immunisation system is the backbone of measles elimination efforts. Equally important is the presence of a sensitive surveillance system that can detect and confirm suspected measles cases, and guide the immunisation program towards swift action to limit the spread of disease. Previous studies have investigated the relationship between vaccination coverage and the incidence of measles ${ }^{1,2}$.

Countries in the WHO African Region have been engaged in 
measles mortality reduction activities since 2011 by implementing WHO recommended strategies ${ }^{3}$. In 2011, the Member States of the WHO African Region adopted a measles elimination goal to be reached by the end of 2020 with the following targets: Measles incidence of less than 1 case per million population at national level; at least 95\% measles immunization coverage at national level and in all districts; minimum 95\% coverage in all measles SIAs; at least $80 \%$ of districts investigating one or more suspected measles cases within a year; and a non-measles febrile rash illness rate of at least 2 per 100000 population at national level $^{4}$. Through the implementation of these strategies, the Region has documented $85 \%$ reduction in estimated measles deaths between 2000 and $2015^{5}$.

Measles elimination is defined as the absence of endemic measles virus transmission in a defined geographic area (e.g., region or country) for $\geq 12$ months in the presence of a well-performing surveillance system. The attainment of measles elimination needs to be documented and independently verified in a structured manner according to the WHO framework for verification of measles elimination. The framework indicates that the achievement of measles and/or rubella elimination should be verified following a standardised process. In addition to the eventual verification of elimination, the process is expected to help countries to monitor progress towards elimination ${ }^{6}$.

This article looks at the overall level of implementation of measles elimination strategies in countries that have maintained more than $90 \%$ first dose measles vaccination coverage (MCV1) for the most recent 5 years for which data is available, and their readiness for verification of elimination.

\section{Methods}

WHO and the United Nations Children's Fund (UNICEF) use data from administrative records reported annually by Member States and from surveys to produce national coverage estimates with first dose measles (MCV1) and the second dose of measles (MCV2) through routine immunization services ${ }^{7}$. Supplemental immunization activities (SIAs) generally are carried as initial, nationwide catch-up SIA targeting all children aged 9 months-14 years, with the goal of eliminating susceptibility to measles in the childhood population; and as periodic follow-up SIAs targeting all children born since the last SIA. Follow-up SIAs are often conducted nationwide every 2-4 years, and focus on children aged 9-59 months, in order to eliminate any measles susceptibility in recent birth cohorts and to protect children who did not sero-convert following the receipt of routine doses of measles vaccine. The target age range for follow-up SIAs may be widened to include older children based on the measles susceptibility pattern in countries.
SIA administrative coverage at the subnational and national level is calculated by tallying the number of administered doses and dividing by the target population. Target population figures are often projections from census data. Countries often conduct post-SIA coverage surveys to validate the administrative vaccination coverage achieved during the SIA $^{8}$.

Countries report the number of measles cases annually to WHO and UNICEF using data either casebased or aggregate surveillance systems. Effective measles surveillance includes case-based surveillance with laboratory testing to confirm cases. In case-based surveillance system, each suspected measles case is investigated. A suspected measles case is defined as a case of generalized maculo-papular rash and fever plus one of the following: cough, runny nose, or conjunctivitis. For each suspected measles case, an individual case investigation form is completed and a blood specimen collected and sent to the national laboratory for testing for measles-specific immunoglobulin M (IgM) antibody ${ }^{9}$.

The surveillance performance of is regularly monitored using standard indicators which look at the sensitivity of the case detection, and also the geographic spread of case notification and investigation. Among the indicators in use, two are considered as the principal monitoring indicators: Non-measles febrile rash illness rate (target of at least 2 per 100000 population) and the proportion of districts that have investigated at least one suspected case of measles with blood specimen per year (target of $80 \%$ or more per year).

We analysed the WHO UNICEF estimates of national immunisation coverage (WUENIC) data for the first measles vaccination dose (MCV1) and identified 11 countries that have maintained more than $90 \%$ MCV1 coverage for the most recent 5 years for which data is available (2011 2015). Subsequent analysis focused on these 11 countries, and included analysis of MCV2 coverage, as well as the coverage data from supplemental immunisation activities (SIAs) conducted in the last 10 years (2007 - 2016). We also reviewed the case-based surveillance data and surveillance performance from the countries for the most recent 5 years period (2012 - 2016).

\section{Results}

We identified 11 countries (Algeria, Botswana, Burundi, Cape Verde, Gambia, Lesotho, Mauritius, Rwanda, Sao Tome and Principe, Seychelles and Tanzania) that had MCV1 coverage of at least $90 \%$ (according to the WUENIC) in the 5 years between 2011 and 2015. Of these countries, 9 countries give MCV1 at 9 months of age, while Mauritius has a 12 months schedule and Seychelles provides MCV1 at 15 months of age. All of these countries have MCV2 in their routine immunisation schedule, provided in the second 
year of life in 8 countries. Algeria and Seychelles give it at 6 years of age, and Mauritius has a 5 years schedule. As of May 2017, Algeria, Burundi and Lesotho use monovalent measles vaccine while the other countries use MeaslesRubella (Botswana, Rwanda, Gambia, Tanzania, Sao Tome and Principe) or Measles-Mumps-Rubella vaccine (Cape Verde, Mauritius and Seychelles).

According to the WUENIC, 7 out of the 11 countries have coverage of $95 \%$ or more in 2015 . Algeria, Botswana,

Table 1. MCV1 and MCV2 coverage. 2011 - 2015. WHO UNICEF coverage estimates.

\section{MCV1 coverage $\quad$ MCV2 coverage}

2011201220132014201520112012201320142015

\begin{tabular}{|l|l|l|l|l|l|l|l|l|l|l|}
\hline Algeria & $95 \%$ & $95 \%$ & $95 \%$ & $95 \%$ & $95 \%$ & $96 \%$ & $95 \%$ & $93 \%$ & $99 \%$ & $99 \%$ \\
\hline
\end{tabular}

\begin{tabular}{|l|l|l|l|l|l|l|l|l|l|l|}
\hline Botswana & $97 \%$ & $97 \%$ & $97 \%$ & $97 \%$ & $97 \%$ & - & - & $83 \%$ & $85 \%$ & $85 \%$ \\
\hline
\end{tabular}

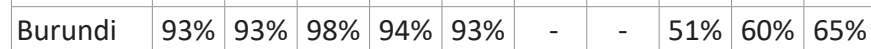

\begin{tabular}{|l|l|l|l|l|l|l|l|l|l|l|}
\hline Cape & $96 \%$ & $91 \%$ & $91 \%$ & $93 \%$ & $92 \%$ & $99 \%$ & $94 \%$ & $89 \%$ & $79 \%$ & $95 \%$ \\
\hline
\end{tabular}

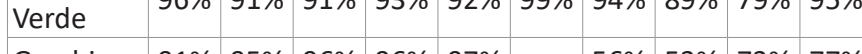

\begin{tabular}{|l|l|l|l|l|l|l|l|l|l|l|}
\hline Gambia & $91 \%$ & $95 \%$ & $96 \%$ & $96 \%$ & $97 \%$ & - & $56 \%$ & $53 \%$ & $73 \%$ & $77 \%$ \\
\hline
\end{tabular}

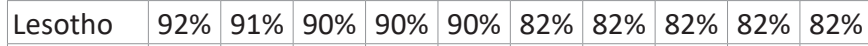

\begin{tabular}{|l|l|l|l|l|l|l|l|l|l|l|l|}
\hline Mauritius & $99 \%$ & $99 \%$ & $99 \%$ & $98 \%$ & $99 \%$ & $89 \%$ & $90 \%$ & $85 \%$ & $85 \%$ & $85 \%$ \\
\hline
\end{tabular}

\begin{tabular}{|l|l|l|l|l|l|l|l|l|l|l|}
\hline Rwanda & $95 \%$ & $97 \%$ & $95 \%$ & $97 \%$ & $97 \%$ & - & - & - & - & $87 \%$ \\
\hline
\end{tabular}

\begin{tabular}{|l|l|l|l|l|l|l|l|l|l|l|}
\hline $\begin{array}{l}\text { Sao Tome } \\
\text { \& Principe }\end{array}$ & $91 \%$ & $92 \%$ & $91 \%$ & $92 \%$ & $93 \%$ & - & - & - & $71 \%$ & $76 \%$ \\
\hline
\end{tabular}

\begin{tabular}{|l|l|l|l|l|l|l|l|l|l|l|l|l|l|l}
\hline Seychelles & $99 \%$ & $98 \%$ & $97 \%$ & $99 \%$ & $98 \%$ & $99 \%$ & $99 \%$ & $97 \%$ & $98 \%$ & $98 \%$ \\
\hline
\end{tabular}

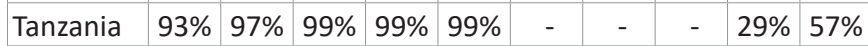

Gambia, Mauritius, Rwanda, Seychelles have maintained coverage of $95 \%$ or more since 2011. (Table 1) The average MCV1 coverage between 2006 - 2015 range from 91\% in Sao Tome and Principe to $99 \%$ in Mauritius.

Six of the 11 countries introduced MCV2 within the past 5 years. In 2015, only Algeria, Cape Verde and Seychelles had coverage of $95 \%$ or more for MCV2. Sao Tome \& Principe and Tanzania started reporting MCV2 since 2014, and Rwanda since 2015. The drop-out rates between MCV1 and MCV2 in 2015 is more than 20\% in Burundi, Gambia and Tanzania, and between 10 and 19\% in Botswana, Mauritius, and Sao Tome and Principe (Table 1).

The measles or measles-rubella (MR) SIAs conducted in the last 10 years in these countries are tabulated in Table 2 . Of the 22 SIAs among the 11 countries, only 6 had administrative coverage of less than $95 \%$. Coverage surveys were done in only 5 of the SIAs. Algeria, Mauritius and Seychelles did not do any nationwide measles or measlesrubella SIAs (Table 2).

As of 2016, these 11 countries, with the exception of Sao Tome and Principe, each have a national measles serological confirmatory laboratory that is part of the African Regional measles-rubella laboratory network. Mauritius, Sao Tome and Principe, and Seychelles have health facility based surveillance systems which include notification of measles

Table 2. Timing and coverage of Measles/ Measles -rubella SIAs. (2007 - 2016)

\begin{tabular}{|c|c|c|c|c|c|c|c|}
\hline COUNTRY & Year & Type of SIAs & $\begin{array}{c}\text { Age group of children } \\
\text { targeted }\end{array}$ & $\begin{array}{l}\text { Number } \\
\text { of children } \\
\text { vaccinated }\end{array}$ & $\begin{array}{l}\text { Coverage } \\
\text { (percent of } \\
\text { target) }\end{array}$ & $\begin{array}{c}\% \text { districts } \\
\text { with } 95 \% \\
\text { or above } \\
\text { coverage }\end{array}$ & $\begin{array}{c}\text { coverage } \\
\text { survey result }\end{array}$ \\
\hline \multirow{3}{*}{ Botswana } & 2009 & Measles Follow-up & 9 - 59 months & 187,380 & $111 \%$ & & \\
\hline & 2013 & Measles Follow-up & 9 - 59 months & 198,341 & $95 \%$ & $54.0 \%$ & \\
\hline & 2016 & MR catch up & $9 \mathrm{mo}-14 \mathrm{yrs}$ & 674,150 & $95 \%$ & $66.7 \%$ & \\
\hline \multirow{3}{*}{ Burundi } & 2009 & Measles Follow-up & $9-59$ months & $1,321,915$ & $95 \%$ & & \\
\hline & 2010 & outbreak response & 9 mo - 14 years & 446,775 & $94 \%$ & & \\
\hline & 2012 & Measles Follow-up & 9 - 59 months & $1,459,304$ & $103 \%$ & $82.2 \%$ & \\
\hline \multirow{2}{*}{ Cape Verde } & 2009 & Measles Follow-up & $9-59$ months & 47,667 & $87 \%$ & & \\
\hline & 2013 & MR catch-up & $9 \mathrm{mo}-24 \mathrm{yrs}$ & 240,166 & $95 \%$ & $45.5 \%$ & \\
\hline \multirow{3}{*}{ Gambia } & 2007 & Measles Follow-up & 9 - 59 months & 241,214 & $96 \%$ & & \\
\hline & 2011 & Measles Follow-up & 9 - 59 months & 294,579 & $95 \%$ & $52.5 \%$ & \\
\hline & 2016 & MR catch up & $9 \mathrm{mo}-14 \mathrm{yrs}$ & 779,654 & $97 \%$ & $85.7 \%$ & \\
\hline \multirow{3}{*}{ Lesotho } & 2007 & Measles Follow-up & 9 - 59 months & 196,490 & $92 \%$ & & \\
\hline & 2010 & Measles Follow-up & $6 \mathrm{mo}-14$ years & 558,335 & $91 \%$ & $40.0 \%$ & $94.3 \%$ \\
\hline & 2013 & Measles Follow-up & 9 - 59 months & 147,676 & $72.7 \%$ & $90.0 \%$ & $92.0 \%$ \\
\hline \multirow{2}{*}{ Rwanda } & 2009 & Measles Follow-up & 9 - 59 months & $1,350,125$ & $101 \%$ & & \\
\hline & 2013 & MR catch-up & 9 mo - 14 years & $4,391,081$ & $103 \%$ & $90.0 \%$ & $98.0 \%$ \\
\hline \multirow{3}{*}{$\begin{array}{l}\text { Saotome \& } \\
\text { Principe }\end{array}$} & 2007 & Measles catch-up & 9 mo - 14 years & 64,487 & $101 \%$ & & \\
\hline & 2012 & Measles Follow-up & 9 - 59 months & 22,528 & $105.4 \%$ & $100.0 \%$ & \\
\hline & 2016 & MR catch up & $9 \mathrm{mo}-14 \mathrm{yrs}$ & 77,285 & $107 \%$ & $100.0 \%$ & \\
\hline \multirow{3}{*}{ Tanzania } & 2008 & Measles Follow-up & 9 - 59 months & $10,826,519$ & $86 \%$ & & \\
\hline & 2011 & Measles Follow-up & 9 - 59 months & $6,686,663$ & $97 \%$ & $60.0 \%$ & $91.6 \%$ \\
\hline & 2014 & MR catch-up & $9 \mathrm{mo}-14 \mathrm{yrs}$ & $20,529,629$ & $97 \%$ & $59.3 \%$ & $89.0 \%$ \\
\hline
\end{tabular}


cases, but do not do case based surveillance for measles and rubella using the WHO AFRO protocol. The countries report cases to WHO and UNICEF through the annual WHOUNICEF joint reporting form (JRF reporting tool), and do not generate indicators for measles case based surveillance performance monitoring as per the WHO AFRO protocol. As of the end of 2016, Rwanda and Lesotho are the only two countries that have attained the performance targets for the two principal measles surveillance monitoring indicators in all the five years, while Botswana and the Gambia have met both targets for at least 4 of the 5 years between 2011 - 2015. In 2015, three of the 8 countries had non measles febrile rash illness rates in excess of 4 per million population. Two countries had similarly high rates in 2016 (Table 3).

Table 3. Measles surveillance performance 2012 - 2016.

\begin{tabular}{|l|c|c|c|c|c|c|c|c|c|c|c|}
\hline & \multicolumn{3}{|c|}{$\begin{array}{c}\text { Non-measles Febrile Rash } \\
\text { Illness Rate per 100,000 } \\
\text { population by year } \\
\text { (target } \geq 2: 100,000)\end{array}$} & \multicolumn{3}{c|}{$\begin{array}{c}\text { Proportion of districts reporting } \\
\text { at least 1 case with blood } \\
\text { specimen by year } \\
\text { (target } \geq 80 \%)\end{array}$} \\
\hline & $\mathbf{2 0 1 2}$ & $\mathbf{2 0 1 3}$ & $\mathbf{2 0 1 4}$ & $\mathbf{2 0 1 5}$ & $\mathbf{2 0 1 6}$ & $\mathbf{2 0 1 2}$ & $\mathbf{2 0 1 3}$ & $\mathbf{2 0 1 4}$ & $\mathbf{2 0 1 5}$ & $\mathbf{2 0 1 6}$ \\
\hline Algeria & 0.1 & 0.2 & 0.1 & 0.1 & 0.1 & $38 \%$ & $31 \%$ & $40 \%$ & $42 \%$ & $42 \%$ \\
\hline Botswana & 4 & 14.9 & 39 & 6.9 & 6.3 & $72 \%$ & $88 \%$ & $96 \%$ & $84 \%$ & $84 \%$ \\
\hline Burundi & 0.6 & 0.4 & 1.8 & 1.3 & 1.3 & $66 \%$ & $36 \%$ & $64 \%$ & $69 \%$ & $69 \%$ \\
\hline $\begin{array}{l}\text { Cape } \\
\text { Verde }\end{array}$ & 0.2 & 0 & 0 & 0 & 0 & $6 \%$ & $6 \%$ & $0 \%$ & $0 \%$ & $6 \%$ \\
\hline Gambia & 4.4 & 7.5 & 5.3 & 4 & 0.9 & $100 \%$ & $83 \%$ & $100 \%$ & $78 \%$ & $56 \%$ \\
\hline Lesotho & 9.4 & 20.4 & 27 & 14.1 & 3.2 & $90 \%$ & $100 \%$ & $100 \%$ & $90 \%$ & $90 \%$ \\
\hline Rwanda & 6.5 & 6.4 & 3.7 & 3.6 & 7.3 & $100 \%$ & $100 \%$ & $94 \%$ & $94 \%$ & $94 \%$ \\
\hline Tanzania & 1.2 & 0.1 & 1.7 & 0.8 & 1.9 & $94 \%$ & $81 \%$ & $100 \%$ & $99 \%$ & $100 \%$ \\
\hline
\end{tabular}

The incidence of confirmed measles (confirmed by lab, epidemiological linkage and clinically) is displayed in Table 4. Gambia and Lesotho had incidence levels of more than 5 confirmed cases per million population in 2016, while Botswana, Cape Verde and Tanzania have attained the elimination target of $<1$ confirmed measles case per million (Table 4).

\section{Discussion}

These 11 countries have maintained very high MCV1 coverage for at least 5 years, and have all introduced MCV2 in the routine immunisation schedule. With the exception of Algeria and Seychelles, the MCV2 coverage remains below the levels required to attain herd immunity and maintain measles elimination. Eight countries have been conducting periodic measles SIAs to close immunity gaps. However, not all countries have been conducting post-campaign coverage surveys to validate administrative coverage.

These 11 countries have maintained low incidence of measles as compared to the Regional average ${ }^{10}$. However, Gambia, Lesotho and Rwanda reported incidence rates of 5 or more per million population in 2016 . The quality of casebased measles surveillance has been consistently below the targets in Algeria, Burundi, Cape Verde and Tanzania.

The experience from various countries shows that to bring down and sustain the incidence at less than 1 per million population and undertake verification of elimination, countries will need to maintain high population immunity to measles; increase the sensitivity of the measles surveillance system, including the characterisation of measles virus genotypes ${ }^{11-13}$. Moreover, all countries should develop plans to be able to detect and rapidly respond to measles importations, which can easily occur across international borders. For example, Rwanda, Burundi and Tanzania share borders with DR Congo, which has been experiencing large measles outbreaks over the past years ${ }^{10,14-15}$. Even after a significant reduction of measles incidence, countries may experience resurgence or importations of measles virus which requires that sensitive disease surveillance and monitoring of circulating viral genotype be in place beforehand in order to detect, document and respond to these situations ${ }^{16-18}$.

Table 4. Number and incidence of confirmed measles cases. 2012 - 2016.

\begin{tabular}{|c|c|c|c|c|c|c|c|c|c|c|c|}
\hline & \multirow{2}{*}{$\begin{array}{l}\text { Total population in } \\
\text { millions as of } 2016\end{array}$} & \multicolumn{5}{|c|}{ Total number of confirmed measles cases } & \multicolumn{5}{|c|}{$\begin{array}{l}\text { Incidence rate of confirmed measles per million } \\
\text { population }\end{array}$} \\
\hline & & 2012 & 2013 & 2014 & 2015 & 2016 & 2012 & 2013 & 2014 & 2015 & 2016 \\
\hline Algeria & 39.5 & 6 & 0 & 0 & 62 & 46 & 0.2 & 1.6 & 0 & 1.6 & 1.2 \\
\hline Botswana & 2.1 & 4 & 1 & 88 & 2 & 1 & 2 & 1 & 41.1 & 1 & 0.5 \\
\hline Burundi & 9.6 & 49 & 0 & 5 & 9 & 13 & 5.5 & 0.9 & 0.5 & 0.9 & 1.4 \\
\hline Cape Verde & 0.5 & 0 & 0 & 0 & 0 & 0 & 0 & 0 & 0 & 0 & 0 \\
\hline Gambia & 1.9 & 0 & 1 & 2 & 23 & 40 & 0 & 13.2 & 1.1 & 13.2 & 21.4 \\
\hline Lesotho & 1.9 & 0 & 1 & 0 & 1 & 13 & 0 & 0.5 & 0 & 0.5 & 6.7 \\
\hline Rwanda & 11 & 81 & 17 & 5 & 1 & 58 & 7.9 & 0.1 & 0.5 & 0.1 & 5 \\
\hline Tanzania & 50.1 & 736 & 21 & 62 & 18 & 36 & 16.4 & 0.4 & 1.3 & 0.4 & 0.7 \\
\hline Mauritius* & 1.3 & 0 & 0 & 0 & - & - & & & & & \\
\hline Seychelles* & 0.1 & 0 & 0 & 0 & 0 & - & & & & & \\
\hline Sao tome \& Principe* & 0.2 & 0 & 0 & 0 & 0 & - & & & & & \\
\hline
\end{tabular}

* Number of cases officially reported to WHO-UNICEF through the JRF. 
Mauritius, Sao Tome and Principe, and Seychelles have not yet established case-based surveillance for measles, but they report aggregate number of cases to WHO and UNICEF through the annual joint reporting tool. Moreover, they do not generate indicators for measles surveillance quality as per the WHO protocol. These countries should include the core variables into their systems in order to be able to monitor the quality of surveillance, measure their performance using the standard indicators, and generate evidence for the verification of measles elimination.

The WHO framework for the verification of measles elimination requires that countries generate and document programmatic evidence related to population immunity, surveillance quality and disease incidence. In addition, the lines of evidence required for the verification exercise include vaccination program sustainability, and genotyping data $^{6}$.

Despite the high routine immunisation coverage, the eleven countries have some program gaps and do not meet all the criteria for the verification of elimination. However, these countries should set up national verification committees as per the WHO protocol in order to initiate the documentation and monitoring of progress, and to address programmatic gaps in the coming years.

There are limitations to this analysis. The coverage figures quoted in this report are from 2015. The WHO UNICEF national coverage estimates for 2016 are not yet available. The reported burden of measles may be underestimated, because not all persons with suspected measles seek care and not all of those who seek care are reported. Data from aggregate reporting systems was not included in this analysis, except for the reports from Mauritius, Seychelles and Sao Tome and Principe. The performance of the national measles laboratories is not included in this analysis.

\section{Conflict of Interest}

None

\section{References}

1. Balcha G Masresha, Amadou Fall, Messeret Eshetu, et al. Measles Mortality Reduction and Pre-Elimination in the African Region, 20012009. J Infect Dis. 2011; 204 (Suppl 1): 198 - 204.

2. Robert Hall, Damien Jolley. International Measles Incidence and immunisation coverage. J Infect Dis. 2011; 204 (Suppl 1).
3. World Health Organization. Progress towards measles control in WHO's African Region, 2001-2008. Wkly Epidemiol Rec. 2009; 84: 397-404.

4. WHO Regional Committee for Africa. Measles Elimination by 2020: A Strategy for the African Region. AFR/RC61/R1. Available at: http://www.afro.who.int/en/sixty-first-session.html Last accessed February 2017.

5. M Patel, M Gacic-Dobo, P Strebel, et al. Progress towards regional measles elimination - worldwide, 2000-2015. Wkly Epidemiol Rec. 2016; 91: 525-536.

6. World Health Organization. Framework for verifying elimination of measles and rubella. Wkly Epidemiol Rec. 2013; 88: 89-99.

7. World Health Organization. WHO/UNICEF estimates of national immunization coverage, WHO-UNICEF estimates of MCV coverage. http://www.who.int/immunization_monitoring/routine/ immunization_coverage/en/index.html Accessed 1 March 2017.

8. World Health Organization. Regional Office for Africa. Measles SIAs Planning \& Implementation Field Guide. Revised April 2010.

9. World Health Organization. Regional Office for Africa. African Regional guidelines for measles and rubella surveillance- Revised April 2015. Available at http://www.afro.who.int/index.php?option=com docman\&task=doc_download\&gid=10814\&Itemid=2593 , Last accessed 17 April 2017.

10. Masresha BG, Dixon MG, Kriss JL, et al. Progress towards measles elimination - African Region, 2013-2016. Wkly Epidemiol Rec. 2017; 92: 229-239.

11. Zahraei SM, Gouya MM, Azad TM, et al. Successful Control and Impending Elimination of Measles in the Islamic Republic of Iran. J Infect Dis. 2011; 204: S305-S311.

12. Gidding HF, Martin NV, Stambos V, et al. Verification of measles elimination in Australia: Application of World Health Organization regional guidelines. J Epidemiol Glob Health. 2016; 6: 197- 209.

13. Khanal S, Sedai TR, Choudary GR, et al. Progress Toward Measles Elimination - Nepal, 2007-2014. MMWR Morb Mortal Wkly Rep. 2016; 65: $206-210$.

14. Scobie HM, Ilunga BK, Mulumba A, et al. Antecedent causes of a measles resurgence in the Democratic Republic of the Congo, Pan Af Med J. 2015; 21: 30. doi:10.11604/pamj. 2015.21.30.6335.

15. World Health Organization. Global progress towards regional measles elimination, worldwide, 2000-2013. Wkly Epidemiol Rec. 2014; 89: 509-516.

16. Ma C, Hao L, Zhang Y, et al. Monitoring progress towards the elimination of measles in China: an analysis of measles surveillance data. Bull World Health Organ. 2014; 92: 340-347.

17. Shibeshi ME, Masresha BG, Smit SB, et al. Measles resurgence in southern Africa: Challenges to measles elimination. Vaccine. 2014: 32: 1798-1807.

18. Fiebelkorn AP, Redd SB, Gastañaduy PA, et al. A Comparison of Postelimination Measles Epidemiology in the United States, 2009-2014 Versus 2001-2008. J Pediatric Infect Dis Soc. 2017; 6 (1): 40-48. 